\title{
Network based statistical analysis detects changes induced by continuous theta-burst stimulation on brain activity
}

\section{at rest}

\section{Chiara Mastropasqua ${ }^{1,2}$, Marco Bozzali ${ }^{1}$, Viviana Ponzo ${ }^{3}$, Giovanni Giulietti ${ }^{1}$, Carlo Caltagirone ${ }^{3,4}$, Mara Cercignani ${ }^{1,5}$ and Giacomo Koch ${ }^{3,4}$ *}

\author{
${ }^{1}$ Neuroimaging Laboratory, IRCCS Santa Lucia, Rome, Italy \\ 2 Department of Neuroscience, Trieste University, Trieste, Italy \\ ${ }^{3}$ Department of Clinical and Behavioural Neurology, IRCCS Santa Lucia, Rome, Italy \\ ${ }^{4}$ Department of Neuroscience, University of Rome Tor Vergata, Rome, Italy \\ ${ }^{5}$ Clinical Imaging Sciences Centre, Brighton and Sussex Medical School, University of Sussex, Falmer, UK
}

\section{Edited by:}

Matteo Feurra, University of Siena, Italy

\section{Reviewed by:}

Miguel Fernandez-Del-Olmo, Universidade da Coruña, Spain

Maria Concetta Pellicciari, IRCCS

Centro San Giovanni di Dio

Fatebenefratelli, Italy

*Correspondence:

Giacomo Koch, Department of Clinical and Behavioural Neurology, IRCCS

Santa Lucia, Rome 00179, Italy e-mail:g.koch@hsantalucia.it
We combined continuous theta-burst stimulation (cTBS) and resting state (RS)-fMRI approaches to investigate changes in functional connectivity $(F C)$ induced by right dorsolateral prefrontal cortex (DLPFC)-cTBS at rest in a group of healthy subjects. Seed-based $\mathrm{fMRI}$ analysis revealed a specific pattern of correlation between the right prefrontal cortex and several brain regions: based on these results, we defined a 29-node network to assess changes in each network connection before and after, respectively, DLPFC-cTBS and sham sessions. A decrease of correlation between the right prefrontal cortex and right parietal cortex (Brodmann areas 46 and 40, respectively) was detected after cTBS, while no significant result was found when analyzing sham-session data. To our knowledge, this is the first study that demonstrates within-subject changes in FC induced by cTBS applied on prefrontal area. The possibility to induce selective changes in a specific region without interfering with functionally correlated area could have several implications for the study of functional properties of the brain, and for the emerging therapeutic strategies based on transcranial stimulation.

Keywords: functional connectivity, cTBS, resting state fMRI, dorsolateral prefrontal cortex, fronto-parietal network

\section{INTRODUCTION}

Brain connectivity has been non-invasively assessed in human subjects using techniques focused on three general network properties: anatomical connectivity, functional connectivity (FC), and response to perturbation/stimulation (1).

Resting state (RS) fMRI is becoming one of the most popular techniques for assessing $\mathrm{FC}$ at rest $(2,3)$, while non-invasive brain stimulation methods can be used to probe how brain connectivity varies in response to an external perturbation. The combination of these techniques holds great promise for addressing important clinical issues (4-6).

Different approaches have been used to investigate the effect of a perturbation on FMRI FC; some studies have been performed to assess the influence of tDCS on RS-fMRI data (7-10). Other work focused on the influence of TMS on task-based effective connectivity (11-14). Just few studies investigated so far the effects of repetitive TMS (rTMS) on RS-fMRI. In one study, rTMS was applied over the left dorsolateral prefrontal cortex (DLPFC), resulting in distal changes of neural activity within the default mode network (DMN) (15). Similarly, two different frequencies of rTMS applied over the left posterior inferior parietal lobule (IPL) were tested to evaluate the effect on the DMN: high-frequency rTMS decreased functional correlations between cortical DMN nodes, but not between these nodes and the hippocampal formation. In contrast, low frequency rTMS increased functional correlations between IPL and the hippocampal formation (16). Another study tested the effects of rTMS on prefrontal-hippocampal coupling during both a working memory task and at rest. Seeded FC analyses demonstrated significant effects of rTMS on the prefrontal network dynamics in the $n$-back task that were not evident during rest (17). All these studies were performed with a strong working hypothesis, either testing only one resting state network (RSN) $(15,16)$ or using coupling analyses within a specified connection (17). Moreover, all of them compared the effects of rTMS in two separate sessions performed on different days, which could have increased the intrinsic variability of the FC measured by fMRI.

Here, for the first time we compared RS-fMRI data recorded before and after real continuous theta-burst repetitive stimulation (cTBS), a powerful protocol, resulting in long-lasting decreases of cortical excitability (18). In contrast with previous studies, we conducted a novel network based statistics (NBSs) (19) approach to include all the most relevant nodes of the areas interconnected with the stimulated site (the right DLPFC). We chose to stimulate the right DLPFC, since TMS of this area is known to modulate several cognitive functions and has a potential role in treating various clinical conditions $(20,21)$.

\section{MATERIALS AND METHODS}

The study was approved by the ethics committee of Santa Lucia Foundation, and written informed consent was obtained from all subjects before study initiation. 


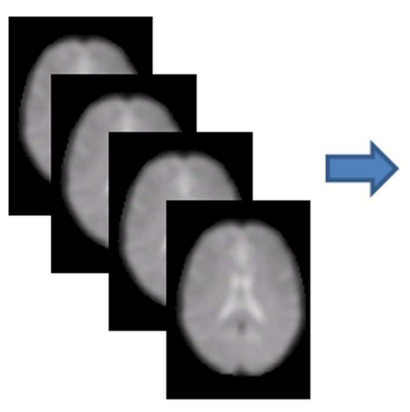

RS

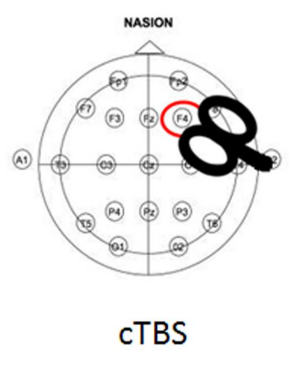

$\square$

cTBS

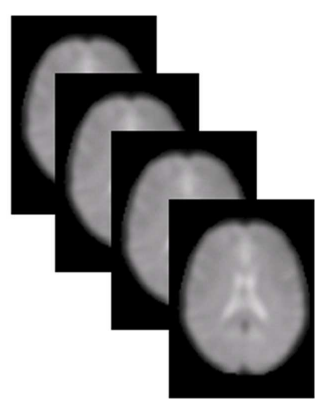

RS

FIGURE 1 | Experimental set up. RS-fMRI was acquired before and after cTBS stimulation in half of the participants.

We recruited 36 healthy volunteers $[m / f=18 / 18$; mean (SD) age $=26.88$ (3.5) years] with no history of medical or psychiatric disorders, autonomic dysfunction, or other major clinical conditions. The experimental session included an MRI scan, followed by either cTBS or sham stimulation, and a post-intervention MRI scan (Figure 1). Each participant was randomly assigned to either group, resulting in 18 participants receiving cTBS, and 18 receiving the sham.

\section{MRI ACOUISITION PROTOCOL}

All imaging was obtained using a head-only $3.0 \mathrm{~T}$ MR scanner (Siemens Magnetom Allegra, Siemens Medical Solutions, Erlangen, Germany). The acquisition protocol included the following sequences: (1) a magnetization-prepared rapid gradient echo (MPRAGE) sequence $(\mathrm{TR}=2500 \mathrm{~ms} ; \mathrm{TE}=2.74 \mathrm{~ms}$; $\mathrm{TI}=900 \mathrm{~ms}$; flip angle $=8^{\circ}$; matrix $=256 \times 208 \times 176$; slab thickness $=1 \mathrm{~mm}$; FOV $=256 \mathrm{~mm} \times 208 \mathrm{~mm} \times 176 \mathrm{~mm}$ ). (2) A series of $\mathrm{T}^{*}$ weighted echo planar imaging (EPI) scans, sensitized to blood oxygenation level dependent contrast (BOLD) $(\mathrm{TR}=2080 \mathrm{~ms} ; \mathrm{TE}=30 \mathrm{~ms} ; 32$ axial slices parallel to AC-PC line; matrix $=64 \times 64$; pixel size $=3 \mathrm{~mm} \times 3 \mathrm{~mm}$; slice thickness $=2.5 \mathrm{~mm}$; flip angle $=70^{\circ}$ ) for RS-fMRI. BOLD EPIs were collected during rest for a $7 \mathrm{~min}$ and $20 \mathrm{~s}$ period, resulting in a total of 220 volumes.

\section{cTBS PROTOCOL}

A MagStim Super Rapid magnetic stimulator (MagStim Company, Whitland, Wales, UK), connected with a figure-of-eight coil with a diameter of $90 \mathrm{~mm}$ was used to deliver cTBS over the scalp site corresponding to the right prefrontal cortex (F4 electrode International 10-20 system). The magnetic stimulus had a biphasic waveform with a pulse width of about $300 \mu$ s. Three-pulse bursts at $50 \mathrm{~Hz}$ repeated every $200 \mathrm{~ms}$ for $40 \mathrm{~s}$ were delivered at $80 \%$ of the active motor threshold (AMT) over right DLPFC (600 pulses). AMT was tested over the motor cortex of the right hemisphere. AMT was defined as the lowest intensity that produced MEPs of $>200 \mu \mathrm{V}$ in at least 5 out of 10 trials when the subject made a $10 \%$ of maximum contraction using visual feedback (22).

Dorsolateral prefrontal cortex was targeted using a neuronavigation system (SofTaxic) to precisely position the coil over the cortical site, using individual T1-weighted magnetic resonance imaging volumes as anatomical reference; this technique has been previously described in detail $(23,24)$. The stimulation points were determined before the experiment and were marked on the adherent plastic cap worn by the subject. To target DLPFC, the coil was positioned over the middle of the line separating the anterior and middle thirds of this gyrus, following the algorithm proposed by Mylius and collaborators (25). According to the anatomical data reported by Rajkowska and Goldman-Rakic (26), this target is localized at the junction between BA9 and BA46. This location is in agreement with meta-analyses of neuroimaging studies on working memory $(27,28)$. The center of the coil was positioned tangentially to the skull with the handle pointing backward angled at $45^{\circ}$ (Figure 1). For sham cTBS, the coil was positioned over the same scalp site, but angled away so that no current was induced in the brain.

\section{fMRI PRE-PROCESSING}

The RS-fMRI data were processed using MATLAB R2007B (MathWork, Natick, MA, USA) and SPM8 ${ }^{1}$. The first four volumes of the functional images were discarded for signal equilibrium and adaptation of participant to scanning noise. Next, slice timing and head motion correction were performed. Participants exhibiting head motion of $>2 \mathrm{~mm}$ maximum translation of $2^{\circ}$ rotation throughout the course of scan were excluded. The images were then normalized using the EPI template provided with SPM8.

In-house software was used to remove the global temporal drift using a third order polynomial fit, the realignment parameters, and the signal averaged over whole brain voxels. Data were band-pass filtered (between 0.01 and $0.08 \mathrm{~Hz}$ ).

\section{SEED-BASED CONNECTIVITY ANALYSIS}

Seed-based connectivity analysis (SBA) was performed to identify the cortical areas functionally connected with the stimulated region. For each subject the mean time course (TC) of the right DLPFC was extracted for each subject using the prefrontal cortex region defined in Harvard Oxford atlas ${ }^{2}$, available with FSL.

\footnotetext{
${ }^{1}$ http://www.fil.ion.ucl.ac.uk/spm/software/spm8/

${ }^{2}$ http://www.cma.mgh.harvard.edu/
} 


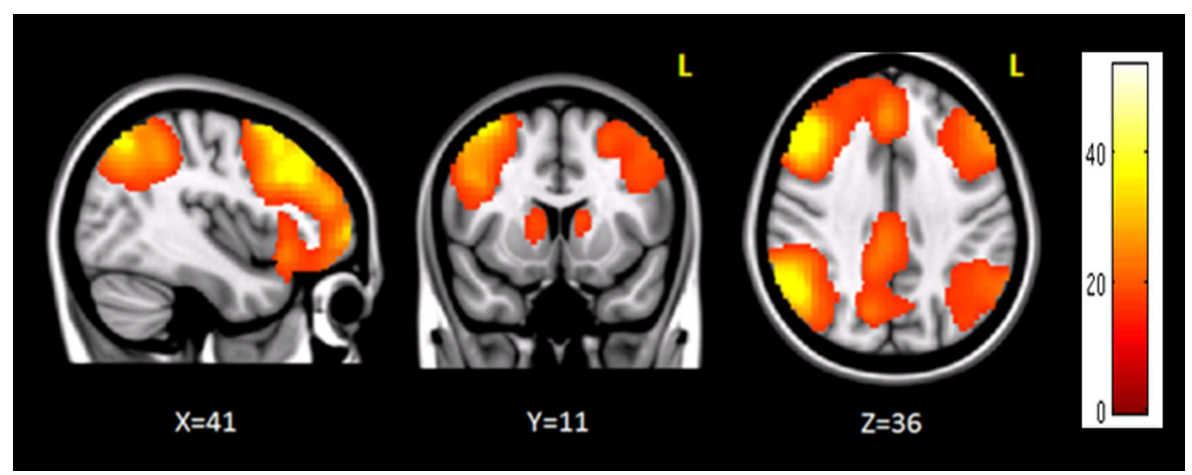

FIGURE 2 | Brain regions functionally correlated to the right prefrontal cortex used as seed in seed-based analysis. The color bar represents the $t$-scores.

Each participant's TC was then used as regressor in a first-level analysis in SPM8, in order to identify the degree of correlation, for every voxel in the brain, with the prefrontal region, adjusting for the motion parameters. Contrast images for positive correlation were fed into a second level analysis using a one-sample $T$-Test. Results were considered significant for $p<0.05$ FWE corrected at voxel level.

\section{NETWORK BASED STATISTIC}

The clusters that resulted to be significantly connected to the right prefrontal cortex (Figure 2) were then defined as the nodes of the network of interest. Using MarsBaR ${ }^{3}$, we created 29 spheres, with a diameter of $8 \mathrm{~mm}$ each (see Table 1; Figure 3), centered at the center of gravity of each of the nodes, from which mean TCs were extracted to estimate a connectivity matrix for each subject. The number of rows and columns in this matrix is the total number of nodes in the network, and the elements are defined as the correlation coefficient between the TC of each pair of nodes.

Once each participant connectivity matrix was obtained, we used the NBS toolbox $(19)^{4}$ to compare the correlation between each node of the network before and after cTBS, using a paired $T$-test design. The false discovery rate (FDR) was used to adjust for multiple comparisons, with 25,000 permutations. Results were considered significant for $p<0.05$. The same analysis was performed on the data acquired before and after sham stimulation.

\section{RESULTS}

Four participants who received sham stimulation were excluded due to excessive motion during fMRI, thus resulting in the following two groups: 18 subjects receiving cTBS $[m / f=9 / 9$; mean (SD) age $=26.72$ (3.8) years] and 14 receiving sham stimulation $[\mathrm{m} / \mathrm{f}=6 / 8$; mean $(\mathrm{SD})$ age $=27.07(3.6)$ years $]$.

Seed-based connectivity analysis revealed a specific pattern of correlation between right DLPFC and several brain regions, including the right and left prefrontal, parietal, temporal cortex, precuneus, posterior cingulated cortex, thalamus, caudate nucleus,

\footnotetext{
${ }^{3}$ http://marsbar.sourceforge.net/

${ }^{4}$ http://www.cmtk.org/viewer/documentation/users/tutorials/tut_nbs.html
}

Table 1 | The table shows the regions used to create the 29-sphere network, their corresponding Brodmann area and MNI coordinates of the center of each sphere.

\begin{tabular}{lllll}
\hline Region & $\begin{array}{l}\text { Brodmann } \\
\text { area }\end{array}$ & $X$ & $Z$
\end{tabular}

Cingulate gyrus (posterior division)

\section{RIGHT}

Frontal pole

BA10

Intracalcarine cortex

Middle temporal gyrus (posterior division)

Paracingulate gyrus (anterior division)

Middle temporal gyrus (temporoccipital part)

Supramarginal gyrus (posterior division)

Frontal pole

Frontal orbital cortex

Precuneous cortex

Middle frontal gyrus

BA17

BA20

\section{BA32}

\section{BA37}

\section{BA40}

BA46

BA47

Caudate nucleus

BA7

BA9

Cruz I (medial cerebellum)

Cruz II (lateral cerebellum)

Thalamus

$\begin{array}{lll}2 & -34 & 40\end{array}$

\section{LEFT}

Frontal pole

Intracalcarine cortex

Middle temporal gyrus (posterior division)

Paracingulate gyrus (anterior division)

Middle temporal gyrus (temporoccipital part)

Supramarginal gyrus (posterior division)

Frontal pole

Frontal orbital cortex

Precuneous cortex

Middle frontal gyrus

Caudate nucleus

Cruz I (medial cerebellum)

Cruz II (lateral cerebellum)

Thalamus

$\begin{array}{lll}32 & 56 & 6\end{array}$

$6-62 \quad 12$

$\begin{array}{lll}60 & -22 \quad 18\end{array}$

$8 \quad 44 \quad 20$

$62-50-10$

$\begin{array}{lll}48 & -44 & 50\end{array}$

$\begin{array}{lll}30 & 50 & 24\end{array}$

$\begin{array}{lll}38 & 22 & -4\end{array}$

$\begin{array}{lll}8 & -66 & 46\end{array}$

$\begin{array}{lll}38 & 24 & 46\end{array}$

$12 \quad 14 \quad 6$

$10-82-28$

$\begin{array}{lll}32 & -72 \quad 30\end{array}$

$8-10 \quad 6$

Every sphere had an $8 \mathrm{~mm}$ radius. 


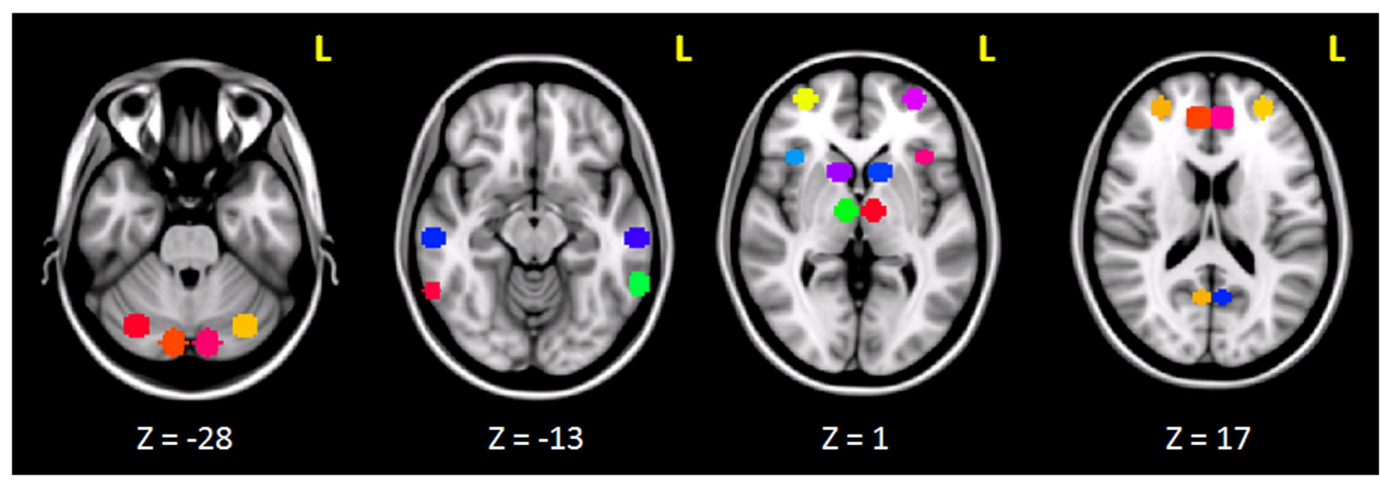

FIGURE 3 | Spherical ROIs (radius $=8 \mathrm{~mm}$ ) defining the nodes of the network investigated before and after cTBS

and cerebellum (Figure 2). The corresponding network nodes are shown in Table 1 and Figure 3. We detected a striking decreased correlation between the right DLPFC and the right posterior parietal cortex (Brodmann areas 46 and 40, respectively) after stimulation $(p<0.05)$ (Figures 4 and $\mathbf{5})$. The same analysis performed on the data acquired before and after sham stimulation did not show any difference among the tested connectivity matrices.

\section{DISCUSSION}

In this study, we provide new evidence for the role of RS-fMRI in detecting changes in brain activity associated with TMS. Through RS-fMRI it is possible to identify functional connections that reflect temporal coupling between distant regions. Thus, characterizing the covariance of the BOLD signal in anatomically distant areas of the brain can be useful to measure the degree to which the network properties are affected by TMS. Here, RS-fMRI was carried out before and immediately after TMS to provide direct measures of the functional organization of the DLPFC-correlated network and its plastic reorganization induced by stimulation.

To assess the influence of the perturbation induced by TMS on FC, we used NBS. It is a novel network based approach to identify functional correlations between different brain regions known to be part of a specific pattern of co-activation. This methodology is based on graph theory, which provides a theoretical framework to examine complex networks, thus revealing important information about their local and global organization $(5,16)$. NBS was used after the identification of a specific network of right DLPFCcorrelated regions, in order to restrict the analysis to the nodes showing functional connections to the stimulation site. Such network was identified using SBA, and it strictly resembles a network previously described as the right fronto-parietal network (FPN) by several groups (5,29-32). Accordingly, we found that DLPFC cTBS induced a selective modulation of the ipsilateral posterior parietal cortex. This finding could be interpreted on the basis of the well known functional interactions strongly linking the activity of the DLPFC with that of the PPC (33). These two areas are jointly implicated in a variety of cognitive functions and are thereby considered two main nodes of the FPN. Indeed, throughout the literature, two strongly lateralized RSNs have consistently been reported, one

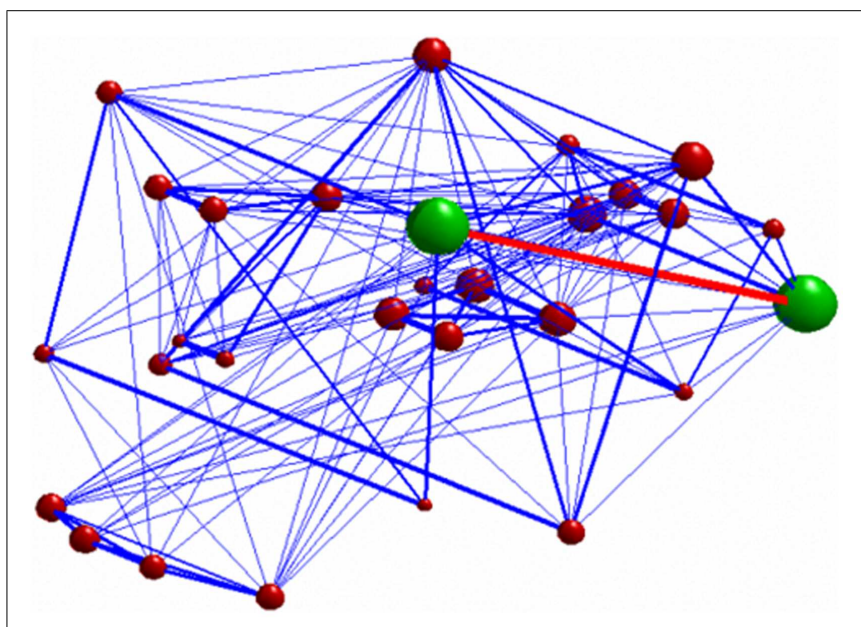

FIGURE 4 | 3D graph representing the investigated network. The green nodes indicate the ROls whose connectivity (represented by the red edge) was decreased after stimulation. The radius of each of the red nodes reflects the node strength (i.e., the sum of the weights of each edge connected to the node). The thickness of the edges reflect the strength of correlation between each node. Only connections with correlation coefficient $>0.3$ are displayed.

predominantly in the right hemisphere and the other in the left hemisphere usually with a specular pattern involving the middle frontal and orbital cortex (BA 6/9/10), the superior parietal cortex (BA 7/40), the middle temporal gyrus (BA 21), and the posterior cingulate cortex (BA 23/31) (30). These two networks are known to be closely coupled in a wide range of cognitive processes, such as working memory, both in adults $(30,34-36)$ and in children and adolescents (37-39), language (40), attention (41-44), and visual processes (45).

Consistently, recent tDCS literature suggests that low-intensity electrical stimulation over the DLPFC can result in transient improvements in a variety of cognitive functions including declarative (46) and working memory $(47,48)$, planning (49), language learning (46), attention (50), and decision making (51).

To better understand the substrate of these changes, the interaction between the nodes of the FPNs has been investigated using 

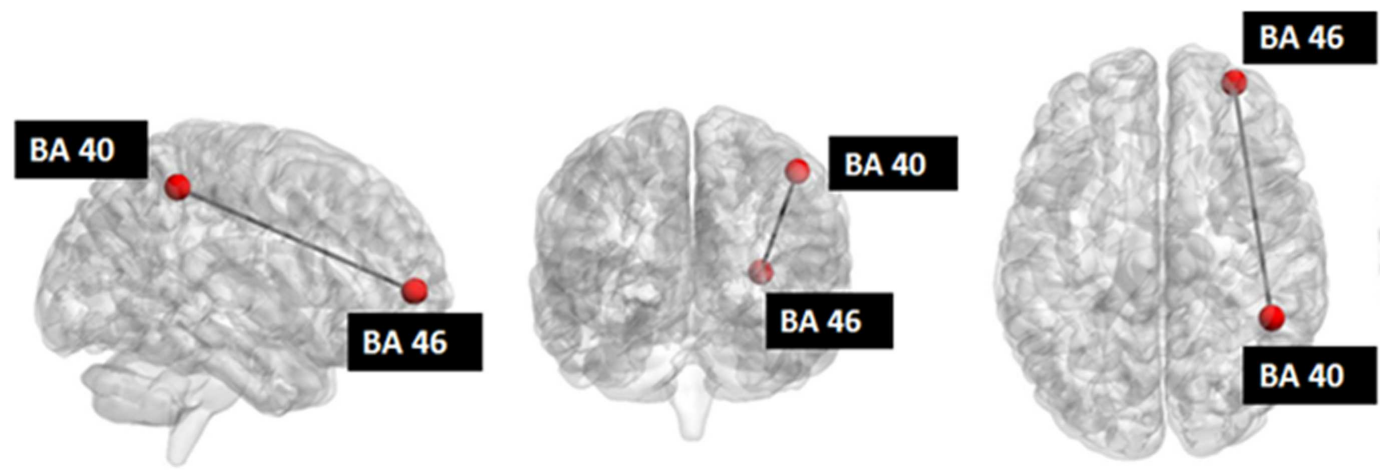

FIGURE 5 | Investigated network: the red nodes indicate the ROls whose connectivity was decreased after stimulation.

tDCS. A previous study (8) examined how active tDCS over the left or right DLPFC in comparison with sham $t D C S$ modulates TC fluctuations within and across the DMN and the anti-correlated network (AN) on RS-fMRI. One of the main results emerging from this work is that active anodal tDCS over the DLPFC results in a stronger temporal FC between prefrontal and parietal regions, supporting our current findings. Similar results were obtained by Keeser et al. (7), who measured significant changes in regional brain connectivity for nodes of the DMN and the right and left FPNs. Such changes were detected after DLPFC-tDCS both, close to the primary stimulation site, and in connected brain regions.

On the other hand, the effects of TMS (as opposed to tDCS) have been mainly evaluated in combination with task-active fMRI, instead of RS-fMRI. Only recently, a number of studies attempted to assess the effect of TMS on FC at rest $(11,13-16,52,53)$. Most of these works were performed with a strong working hypothesis, either testing only one RSN $(15,16)$ or using coupling analyses within a specified connection (17). Crucially, all of them evaluated the effects of rTMS by comparing post-stimulation vs. post-sham data recorded on separate days, introducing a bias due to the intrinsic variability of RS-fMRI. In contrast, in order to reduce the effect of intrinsic individual variability, we compared for the first time RS-fMRI data recorded within the same session before and after TMS, with a short interval between MRI sessions.

Our data indicate a selective influence of right DLPFC-cTBS on the ipsilateral posterior parietal cortex, while no connectivity change was detected after sham stimulation. As it is known that cTBS is able to induce prolonged cortical inhibition (18), the decreased correlation between BA46 and BA40 we observed after stimulation could be explained by two alternative hypotheses: (i) cTBS is able to induce cortical inhibition just in the stimulated site with a consequent disruption of the co-activation of the two areas; (ii) the inhibition of cortical activity occurs immediately in the stimulation site, subsequently spreading to distant connected area. The propagation of inhibitory signal at microscopic level induces a de-synchronization of normal coupling activity of the areas involved. So the de-coupling of neuronal activity we observed through the BOLD signal could reflect an undergoing mechanism of signal propagation. Thus, we hypothesize that
cTBS does not solely produce focal effects by selectively affecting an isolated patch of cortex. Rather, target sites should be considered as nodes within a widespread network of interacting brain regions, where perturbing or boosting processing of one element can also influence several others. We can only speculate on why we found a selective modulation of the DLPFC-PPC connection. The DLPFC and PPC neuronal assemblies have a strong functional coupling that could be more sensible to an external perturbation such as that induced by the low-intensity cTBS protocol applied in the current study (33). However, it is likely that by simply increasing the intensity of the magnetic field or changing the frequency of stimulation it could be possible to affect the coupling among other interconnected nodes. Notably, recent evidence suggests that an individual approach based on FC MRI could provide the most reliable approach to detect the effects of DLPFC TMS $(1,54)$.

Our results could also have several implications for clinical applications, as it has been demonstrated the role of rTMS of the DLPFC in the treatment of major depressive disorder (MDD). This therapeutic effect can be achieved by either excitatory stimulation of the left $(52,55-57)$ or inhibitory stimulation of the right DLPFC (58-60). A recent meta-analysis study conducted by Chen and collaborators (20), demonstrated that, despite the comparable efficacy of both methodology, the latter (inhibitory TMS) may be a more acceptable treatment for MDD than the former (excitatory TMS), based on patients reporting less headaches, and on the decrease risk of inducing adverse events such as seizures (61). The present results could also be important for other conditions in which the non-invasive modulation of the FPN can provide notable clinical improvements, such as the case of post-stroke hemispatial neglect (62).

In conclusion, our findings provide new insights into the mechanisms of stimulation-induced brain plasticity by demonstrating that the network communication at rest shapes the brain reorganization induced by cTBS. The use of TMS and RS-fMRI allows to characterize both local (i.e., in the cortical tissue directly under the TMS coil) and remote (i.e., distant from the original cortical target site) effects of TMS in more detail, leading to a better understanding of TMS-induced modulations in neural processing. 


\section{ACKNOWLEDGMENTS}

The Neuroimaging Laboratory is supported in part by grants from the Italian Ministry of Health.

\section{REFERENCES}

1. Fox MD, Buckner RL, White MP, Greicius MD, Pascual-Leone A. Efficacy of transcranial magnetic stimulation targets for depression is related to intrinsic functional connectivity with the subgenual cingulate. Biol Psychiatry (2012) 72:595-603. doi:10.1016/j.biopsych.2012.04.028

2. Aertsen AM, Gerstein GL. Evaluation of neuronal connectivity: sensitivity of cross-correlation. Brain Res (1985) 340:341-54. doi:10.1016/0006-8993(85) 90931-X

3. Friston KJ, Frith CD, Frackowiak RS. Principal component analysis learning algorithms: a neurobiological analysis. Proc Biol Sci (1993) 254:47-54. doi:10.1098/rspb.1993.0125

4. Fox MD, Raichle ME. Spontaneous fluctuations in brain activity observed with functional magnetic resonance imaging. Nat Rev Neurosci (2007) 8:700-11. doi:10.1038/nrn2201

5. van den Heuvel MP, Hulshoff Pol HE. Exploring the brain network: a review on resting-state fMRI functional connectivity. Eur Neuropsychopharmacol (2010) 20:519-34. doi:10.1016/j.euroneuro.2010.03.008

6. Deco G, Jirsa VK, Mcintosh AR. Emerging concepts for the dynamical organization of resting-state activity in the brain. Nat Rev Neurosci (2011) 12:43-56. doi:10.1038/nrn2961

7. Keeser D, Meindl T, Bor J, Palm U, Pogarell O, Mulert C, et al. Prefrontal transcranial direct current stimulation changes connectivity of resting-state networks during fMRI. J Neurosci (2011) 31:15284-93. doi:10.1523/JNEUROSCI.054211.2011

8. Pena-Gomez C, Sala-Lonch R, Junque C, Clemente IC, Vidal D, Bargallo N, et al. Modulation of large-scale brain networks by transcranial direct current stimulation evidenced by resting-state functional MRI. Brain Stimul (2011) 5:252-63. doi:10.1016/j.brs.2011.08.006

9. Polania R, Paulus W, Nitsche MA. Modulating cortico-striatal and thalamocortical functional connectivity with transcranial direct current stimulation. Hum Brain Mapp (2011) 33:2499-508. doi:10.1002/hbm.21380

10. Meinzer M, Antonenko D, Lindenberg R, Hetzer S, Ulm L, Avirame K, et al. Electrical brain stimulation improves cognitive performance by modulating functional connectivity and task-specific activation. J Neurosci (2012) 32:1859-66. doi:10.1523/JNEUROSCI.4812-11.2012

11. Lee L, Siebner HR, Rowe JB, Rizzo V, Rothwell JC, Frackowiak RS, et al. Acute remapping within the motor system induced by low-frequency repetitive transcranial magnetic stimulation. J Neurosci (2003) 23:5308-18. doi:10. 1002/hbm 20371

12. Pleger B, Blankenburg F, Bestmann S, Ruff CC, Wiech K, Stephan KE, et al. Repetitive transcranial magnetic stimulation-induced changes in sensorimotor coupling parallel improvements of somatosensation in humans. J Neurosci (2006) 26:1945-52. doi:10.1523/JNEUROSCI.4097-05.2006

13. O'Shea J, Johansen-Berg H, Trief D, Gobel S, Rushworth MF. Functionally specific reorganization in human premotor cortex. Neuron (2007) 54:479-90. doi:10.1016/j.neuron.2007.04.021

14. Grefkes C, Eickhoff SB, Nowak DA, Dafotakis M, Fink GR. Dynamic intra- and interhemispheric interactions during unilateral and bilateral hand movements assessed with fMRI and DCM. Neuroimage (2008) 41:1382-94. doi:10.1016/j. neuroimage.2008.03.048

15. van der Werf YD, Sanz-Arigita EJ, Menning S, Van Den Heuvel OA. Modulating spontaneous brain activity using repetitive transcranial magnetic stimulation. BMC Neurosci (2010) 11:145. doi:10.1186/1471-2202-11-145

16. Eldaief MC, Halko MA, Buckner RL, Pascual-Leone A. Transcranial magnetic stimulation modulates the brain's intrinsic activity in a frequency-dependent manner. Proc Natl Acad Sci U S A (2011) 108:21229-34. doi:10.1073/pnas. 1113103109

17. Bilek E, Schafer A, Ochs E, Esslinger C, Zangl M, Plichta MM, et al. Application of high-frequency repetitive transcranial magnetic stimulation to the DLPFC alters human prefrontal-hippocampal functional interaction. J Neurosci (2013) 33:7050-6. doi:10.1523/JNEUROSCI.3081-12.2013

18. Huang YZ, Edwards MJ, Rounis E, Bhatia KP, Rothwell JC. Theta burst stimulation of the human motor cortex. Neuron (2005) 45:201-6. doi:10.1016/j.neuron. 2004.12.033
19. Zalesky A, Fornito A, Bullmore ET. Network-based statistic: identifying differences in brain networks. Neuroimage (2010) 53:1197-207. doi:10.1016/j. neuroimage.2010.06.041

20. Chen J, Zhou C, Wu B, Wang Y, Li Q, Wei Y, et al. Left versus right repetitive transcranial magnetic stimulation in treating major depression: a metaanalysis of randomised controlled trials. Psychiatry Res (2013) 210(3):1260-4. doi:10.1016/j.psychres.2013.09.007

21. George MS, Taylor JJ, Short EB. The expanding evidence base for rTMS treatment of depression. Curr Opin Psychiatry (2013) 26:13-8. doi:10.1097/YCO. 0b013e32835ab46d

22. Rothwell JC. Techniques and mechanisms of action of transcranial stimulation of the human motor cortex. J Neurosci Methods (1997) 74:113-22. doi:10.1016/S0165-0270(97)02242-5

23. Koch G, Oliveri M, Torriero S, Salerno S, Lo Gerfo E, Caltagirone C. Repetitive TMS of cerebellum interferes with millisecond time processing. Exp Brain Res (2007) 179:291-9. doi:10.1007/s00221-006-0791-1

24. Koch G, Oliveri M, Caltagirone C. Neural networks engaged in milliseconds and seconds time processing: evidence from transcranial magnetic stimulation and patients with cortical or subcortical dysfunction. Philos Trans R Soc Lond B Biol Sci (2009) 364:1907-18. doi:10.1098/rstb.2009.0018

25. Mylius V, Ayache SS, Ahdab R, Farhat WH, Zouari HG, Belke M, et al. Definition of DLPFC and M1 according to anatomical landmarks for navigated brain stimulation: inter-rater reliability, accuracy, and influence of gender and age. Neuroimage (2013) 78:224-32. doi:10.1016/j.neuroimage.2013. 03.061

26. Rajkowska G, Goldman-Rakic PS. Cytoarchitectonic definition of prefrontal areas in the normal human cortex: II. Variability in locations of areas 9 and 46 and relationship to the Talairach Coordinate System. Cereb Cortex (1995) 5:323-37. doi:10.1093/cercor/5.4.307

27. Glahn DC, Ragland JD, Abramoff A, Barrett J, Laird AR, Bearden CE, et al. Beyond hypofrontality: a quantitative meta-analysis of functional neuroimaging studies of working memory in schizophrenia. Hum Brain Mapp (2005) 25:60-9. doi:10.1002/hbm.20138

28. Fitzgerald PB, Oxley TJ, Laird AR, Kulkarni J, Egan GF, Daskalakis ZJ. An analysis of functional neuroimaging studies of dorsolateral prefrontal cortical activity in depression. Psychiatry Res (2006) 148:33-45. doi:10.1016/j.pscychresns.2006. 04.006

29. Beckmann CF, DeLuca M, Devlin J, Smith S. Investigations into resting-state connectivity using independent component analysis. Philos Trans R Soc Lond B Biol Sci (2005) 360:1001-13. doi:10.1098/rstb.2005.1634

30. Damoiseaux JS, Rombouts SA, Barkhof F, Scheltens P, Stam CJ, Smith SM, et al. Consistent resting-state networks across healthy subjects. Proc Natl Acad Sci U $S$ A (2006) 103:13848-53. doi:10.1073/pnas.0601417103

31. Calhoun VD, Kiehl KA, Pearlson GD. Modulation of temporally coherent brain networks estimated using ICA at rest and during cognitive tasks. Hum Brain Mapp (2008) 29:828-38. doi:10.1002/hbm.20581

32. Jeong B, Choi J, Kim JW. MRI study on the functional and spatial consistency of resting state-related independent components of the brain network. Korean J Radiol (2012) 13:265-74. doi:10.3348/kjr.2012.13.3.265

33. Koch G, Bozzali M, Bonni S, Giacobbe V, Caltagirone C, Cercignani M. fMRI resting slow fluctuations correlate with the activity of fast cortico-cortical physiological connections. PLoS One (2012) 7:e52660. doi:10.1371/journal. pone. 0052660

34. D'Esposito M, Aguirre GK, Zarahn E, Ballard D, Shin RK, Lease J. Functional MRI studies of spatial and nonspatial working memory. Brain Res Cogn Brain Res (1998) 7:1-13. doi:10.1016/S0926-6410(98)00004-4

35. Wager TD, Smith EE. Neuroimaging studies of working memory: a meta-analysis. Cogn Affect Behav Neurosci (2003) 3:255-74. doi:10.3758/CABN. 3.4.255

36. van Asselen M, Kessels RP, Neggers SF, Kappelle LJ, Frijns CJ, Postma A. Brain areas involved in spatial working memory. Neuropsychologia (2006) 44:1185-94. doi:10.1016/j.neuropsychologia.2005.10.005

37. Kwon H, Reiss AL, Menon V. Neural basis of protracted developmental changes in visuo-spatial working memory. Proc Natl Acad Sci U S A (2002) 99:13336-41. doi:10.1073/pnas.162486399

38. Thomason ME, Race E, Burrows B, Whitfield-Gabrieli S, Glover GH, Gabrieli JD. Development of spatial and verbal working memory capacity in the human brain. J Cogn Neurosci (2009) 21:316-32. doi:10.1162/jocn.2008. 21028 
39. Finn AS, Sheridan MA, Kam CL, Hinshaw S, D'esposito M. Longitudinal evidence for functional specialization of the neural circuit supporting working memory in the human brain. J Neurosci (2010) 30:11062-7. doi:10.1523/ JNEUROSCI.6266-09.2010

40. Smith SM, Fox PT, Miller KL, Glahn DC, Fox PM, Mackay CE, et al. Correspondence of the brain's functional architecture during activation and rest. Proc Natl Acad Sci U S A (2009) 106:13040-5. doi:10.1073/pnas.0905267106

41. Corbetta M, Akbudak E, Conturo TE, Snyder AZ, Ollinger JM, Drury HA, et al. A common network of functional areas for attention and eye movements. Neuron (1998) 21:761-73. doi:10.1016/S0896-6273(00)80593-0

42. Fink GR, Marshall JC, Weiss PH, Zilles K. The neural basis of vertical and horizontal line bisection judgments: an fMRI study of normal volunteers. Neuroimage (2001) 14:S59-67. doi:10.1006/nimg.2001.0819

43. Dosenbach NU, Fair DA, Miezin FM, Cohen AL, Wenger KK, Dosenbach RA, et al. Distinct brain networks for adaptive and stable task control in humans. Proc Natl Acad Sci U S A (2007) 104:11073-8. doi:10.1073/pnas.0704320104

44. Vannini P, Almkvist O, Dierks T, Lehmann C, Wahlund LO. Reduced neuronal efficacy in progressive mild cognitive impairment: a prospective fMRI study on visuospatial processing. Psychiatry Res (2007) 156:43-57. doi:10.1016/ j.pscychresns.2007.02.003

45. De Luca M, Beckmann CF, De Stefano N, Matthews PM, Smith SM. fMRI resting state networks define distinct modes of long-distance interactions in the human brain. Neuroimage (2006) 29:1359-67. doi:10.1016/j.neuroimage.2005. 08.035

46. Floel A, Suttorp W, Kohl O, Kurten J, Lohmann H, Breitenstein C, et al. Noninvasive brain stimulation improves object-location learning in the elderly. Neurobiol Aging (2008) 33:1682-9. doi:10.1016/j.neurobiolaging.2011.05.007

47. Fregni F, Boggio PS, Nitsche M, Bermpohl F, Antal A, Feredoes E, et al. Anodal transcranial direct current stimulation of prefrontal cortex enhances working memory. Exp Brain Res (2005) 166:23-30. doi:10.1007/s00221-005-2334-6

48. Zaehle T, Sandmann P, Thorne JD, Jancke L, Herrmann CS. Transcranial direct current stimulation of the prefrontal cortex modulates working memory performance: combined behavioural and electrophysiological evidence. BMC Neurosci (2011) 12:2. doi:10.1186/1471-2202-12-2

49. Dockery CA, Hueckel-Weng R, Birbaumer N, Plewnia C. Enhancement of planning ability by transcranial direct current stimulation. J Neurosci (2009) 29:7271-7. doi:10.1523/JNEUROSCI.0065-09.2009

50. Boggio PS, Bermpohl F, Vergara AO, Muniz AL, Nahas FH, Leme PB, et al. Gono-go task performance improvement after anodal transcranial DC stimulation of the left dorsolateral prefrontal cortex in major depression. J Affect Disord (2007) 101:91-8. doi:10.1016/j.jad.2006.10.026

51. Boggio PS, Campanha C, Valasek CA, Fecteau S, Pascual-Leone A, Fregni F. Modulation of decision-making in a gambling task in older adults with transcranial direct current stimulation. Eur J Neurosci (2010) 31:593-7. doi:10.1111/j.14609568.2010.07080.x

52. Padberg F, George MS. Repetitive transcranial magnetic stimulation of the prefrontal cortex in depression. Exp Neurol (2009) 219:2-13. doi:10.1016/j. expneurol.2009.04.020

53. Vercammen A, Knegtering H, Liemburg EJ, Den Boer JA, Aleman A. Functional connectivity of the temporo-parietal region in schizophrenia: effects of rTMS treatment of auditory hallucinations. J Psychiatr Res (2010) 44:725-31. doi:10.1016/j.jpsychires.2009.12.011
54. Fox MD, Liu H, Pascual-Leone A. Identification of reproducible individualized targets for treatment of depression with TMS based on intrinsic connectivity. Neuroimage (2012) 66C:151-60. doi:10.1016/j.neuroimage.2012.10.082

55. George MS, Post RM. Daily left prefrontal repetitive transcranial magnetic stimulation for acute treatment of medication-resistant depression. Am J Psychiatry (1995) 168:356-64. doi:10.1176/appi.ajp.2010.10060864

56. Pascual-Leone A, Rubio B, Pallardo F, Catala MD. Rapid-rate transcranial magnetic stimulation of left dorsolateral prefrontal cortex in drug-resistant depression. Lancet (1996) 348:233-7. doi:10.1016/S0140-6736(96)01219-6

57. O'Reardon JP, Solvason HB, Janicak PG, Sampson S, Isenberg KE, Nahas Z, et al. Efficacy and safety of transcranial magnetic stimulation in the acute treatment of major depression: a multisite randomized controlled trial. Biol Psychiatry (2007) 62:1208-16. doi:10.1016/j.biopsych.2007.01.018

58. Isenberg K, Downs D, Pierce K, Svarakic D, Garcia K, Jarvis M, et al. Low frequency rTMS stimulation of the right frontal cortex is as effective as high frequency rTMS stimulation of the left frontal cortex for antidepressant-free, treatment-resistant depressed patients. Ann Clin Psychiatry (2005) 17:153-9. doi:10.1080/10401230591002110

59. Pallanti S, Bernardi S, Di Rollo A, Antonini S, Quercioli L. Unilateral low frequency versus sequential bilateral repetitive transcranial magnetic stimulation: is simpler better for treatment of resistant depression? Neuroscience (2010) 167:323-8. doi:10.1016/j.neuroscience.2010.01.063

60. Rossini D, Lucca A, Magri L, Malaguti A, Smeraldi E, Colombo C, et al. A symptom-specific analysis of the effect of high-frequency left or low-frequency right transcranial magnetic stimulation over the dorsolateral prefrontal cortex in major depression. Neuropsychobiology (2010) 62:91-7. doi:10.1159/000315439

61. Schutter DJ. Quantitative review of the efficacy of slow-frequency magnetic brain stimulation in major depressive disorder. Psychol Med (2010) 40:1789-95. doi:10.1017/S003329171000005X

62. Koch G, Bonni S, Giacobbe V, Bucchi G, Basile B, Lupo F, et al. theta-burst stimulation of the left hemisphere accelerates recovery of hemispatial neglect. Neurology (2012) 78:24-30. doi:10.1212/WNL.0b013e31823ed08f

Conflict of Interest Statement: The authors declare that the research was conducted in the absence of any commercial or financial relationships that could be construed as a potential conflict of interest.

Received: 09 June 2014; paper pending published: 03 July 2014; accepted: 21 July 2014; published online: 05 August 2014.

Citation: Mastropasqua C, Bozzali M, Ponzo V, Giulietti G, Caltagirone C, Cercignani $M$ and Koch $G$ (2014) Network based statistical analysis detects changes induced by continuous theta-burst stimulation on brain activity at rest. Front. Psychiatry 5:97. doi: 10.3389/fpsyt.2014.00097

This article was submitted to Neuropsychiatric Imaging and Stimulation, a section of the journal Frontiers in Psychiatry.

Copyright (C) 2014 Mastropasqua, Bozzali, Ponzo, Giulietti, Caltagirone, Cercignani and Koch. This is an open-access article distributed under the terms of the Creative Commons Attribution License (CC BY). The use, distribution or reproduction in other forums is permitted, provided the original author(s) or licensor are credited and that the original publication in this journal is cited, in accordance with accepted academic practice. No use, distribution or reproduction is permitted which does not comply with these terms. 